\title{
Potencialidades, demandas e fragilidades no atendimento de enfermagem a crianças vítimas de queimaduras: revisão integrativa de literatura
}

\author{
Potential, demands and weaknesses in nursing care for child burn victims: an integrative literature \\ review
}

Potencial, demandas y debilidades en la atención de enfermería para niños víctimas de quemaduras: una revisión integradora de la literatura

\begin{abstract}
Resumo
Objetivo: identificar as evidências científicas nacionais e internacionais as potencialidades, demandas e fragilidades no perfil do atendimento de enfermagem a crianças vítimas de queimaduras. Método: Revisão integrativa da literatura, realizada em quatro bases de dados, com os critérios de inclusão: artigos na íntegra, acesso gratuito, disponível online, idiomas em inglês, espanhol e português. A amostra final foi composta por 10 estudos. Resultados: A partir da leitura e da comparação entre os resultados dos estudos, emergiram quatro categorias, organizadas em: potencialidades, demandas e fragilidades no atendimento a crianças vítimas de queimaduras. Conclusão: Nos estudos selecionados, identificamos potencialidades, fragilidades e, demandas. Ainda que potencialidades, como rede articulada, autonomia na assistência, acolhimento e empatia; existem fragilidades, como falta de estratégias para comunicação efetiva e inadequado registro de informações, que desafiam a concretização do atendimento integral.

Palavras-chave: Queimaduras; Crianças; Enfermagem.
\end{abstract}

\begin{abstract}
Objective: identify national and international scientific evidence on the potential, demands and weaknesses in the profile of nursing care for children who are victims of burns. Method: Integrative literature review, carried out in four databases, with the inclusion criteria: full articles, free access, available online, languages in English, Spanish and Portuguese. The final sample consisted of 10 studies. Results: From the reading and comparison between the results of the studies, four categories emerged, organized into: strengths, demands and weaknesses in caring for children who were victims of burns. Conclusion: In the selected studies, we identified strengths, weaknesses and demands. Although potentials, such as an articulated network, autonomy in care, welcoming and empathy; there are weaknesses, such as lack of effective communication strategies and inadequate recording of information, which challenge the implementation of comprehensive care.
\end{abstract}

Keywords: Burns; Child; Nursing.

\section{Resumen}

Objetivo: identificar evidencia científica nacional e internacional sobre las potencialidades, demandas y debilidades en el perfil del cuidado de enfermería para niños víctimas de quemaduras. Método: Revisión bibliográfica integradora, realizada en cuatro bases de datos, con los criterios de inclusión: artículos completos, acceso gratuito, disponibles en línea, idiomas en inglés, español y portugués. La muestra final consistió en 10 estudios. Resultados: de la lectura y comparación entre los resultados de los estudios surgieron cuatro categorías, organizadas en: fortalezas, demandas y 
debilidades en el cuidado de los niños víctimas de quemaduras. Conclusión: En los estudios seleccionados, identificamos fortalezas, debilidades y demandas. Aunque potenciales, como una red articulada, autonomía en el cuidado, acogida y empatía; existen debilidades, como la falta de estrategias de comunicación efectiva y el registro inadecuado de la información, que desafían la implementación de la atención integral.

Palabras clave: Quemaduras; Niños; Enfermería.

\section{Introdução}

As queimaduras são caracterizadas por danos aos tecidos causados por agentes físicos, químicos, biológicos e / ou radioativos. Esses agentes atingem a pele intacta e podem apresentar variadas extensões e graus de danos na área afetada (Aragão et al., 2012). Assim, segundo a literatura, as queimaduras são classificadas como um grave problema de saúde pública e uma das principais causas de morte registradas no Brasil (Aragão et al., 2012; Prestes et al., 2015).

Epidemiologicamente, as lesões por queimaduras são um dos tipos de trauma mais comuns. Aproximadamente 12 mil pessoas morreram devido a queimaduras, a inalação de ar quente ou fumaça tóxica. Crianças e idosos constituem o principal grupo de risco para casos de queimaduras (Smeltzer; Bare, 2002).

As crianças estão mais sujeitas a acidentes devido à curiosidade e à imaturidade física e cognitiva. Portanto, por essas características os tornarem mais vulneráveis, eles precisam que os adultos estejam mais vigilantes para promover sua proteção e bem-estar (Gurgel; Monteiro, 2016). Queimaduras em crianças menores de 9 anos são assunto abordado na literatura nacional e internacional. Em 2015, as queimaduras acidentais em crianças de 1 a 4 anos foram a quinta causa de morte e ocuparam o terceiro lugar entre as crianças de 5 a 9 anos. Mais da metade das queimaduras infantis são causadas por vapor de água quente (Maciel et al., 2018).

Pontua-se, que as crianças frequentemente são vítimas de queimaduras, sendo responsáveis por $60 \%$ dos casos de acidentes. $\mathrm{O}$ escaldamento é a principal fonte de queimaduras domésticas e são causadas por líquidos superaquecidos, como água, leite, sopa, café, chá e imersão em água. Por outro lado, as queimaduras podem ocorrer devido ao manuseio incorreto de isqueiros, fósforos, fornos elétricos, fogões e fornos de micro-ondas (Nettina, 1999).

Nesse caso, no atendimento ao paciente queimado, devido à reação sistêmica de cada indivíduo, deve-se levar em consideração a idade do cliente para avaliar a gravidade da lesão causada pela queimadura. Principalmente em crianças, a área de superfície corporal desproporcional em relação ao peso pode exacerbar possíveis complicações (Vale, 2005).

Novaes (2003) consta que os cuidados primários para pacientes queimados são essenciais para o sucesso final do tratamento porque ajudam a reduzir a morbilidade e mortalidade. Pacientes queimados devem ser atendidos em caráter emergencial, pois quanto maior a exposição do paciente queimado ao agente causador, maior o risco de complicações como a sepse.

Um atendimento humanizado atende às necessidades integrais dos clientes. O enfermeiro é responsável pelo planejamento desse tipo de cuidado por meio da Sistematização da Assistência de Enfermagem (SAE). Esta ferramenta caracteriza-se por um processo de sistematizado do cuidado baseado em métodos científicos, podendo formular ações para promover a melhoria da qualidade da saúde do indivíduo e intervir na patologia por meio do processo de enfermagem (Truppel et al., 2009).

$\mathrm{O}$ atendimento pediátrico no pronto-socorro requer rápida assistência e resolução efetiva dos casos (Santos et al., 2011). Portanto, a assistência de enfermagem à criança queimada em serviços de urgência e emergência deve seguir protocolos que padronizem o atendimento e minimizem possíveis erros e complicações (Brasil, 2012).

O paciente queimado sofre uma interrupção do seu cotidiano, desencadeando uma série de pensamentos negativos. Cabe à enfermagem saber reconhecer as mudanças de comportamento e proporcionar conforto aos indivíduos e suas famílias 
(Bergamasco et al., 2004). Os pais de crianças vítimas de tais acidentes podem vivenciar diferentes reações e emoções, o que pode afetar negativamente a saúde de crianças acidentadas (Oliveira et al., 2016).

Diante do exposto, justifica-se a realização da presente pesquisa considerando sua importância epidemiológica, já que a maioria dos casos de queimaduras infantis são evitáveis. Por esse motivo, faz-se necessário a realização desta pesquisa para reiterar a importância do cuidado profissional de enfermagem, cujo objetivo consistiu em: identificar as evidências científicas nacionais e internacionais acerca do perfil do atendimento de enfermagem a crianças vítimas de queimaduras.

\section{Metodologia}

Foi realizada uma pesquisa na literatura com intuito de identificar a produção científica a respeito do Perfil do Atendimento de Enfermagem a crianças vítimas de queimaduras.

Para tanto, foram selecionados os descritores no DeCS/Mesh (Descritores em Ciências da Saúde/Medical Subject Headings): queimaduras/ burns, crianças/ kids, em inglês, espanhol e português. As bases de dados selecionadas foram PUBMED/MEDILINE (Via National Library of Medicine), Literatura Latino-Americana e do Caribe em Ciência da Saúde (LILACS) Sciverse Scopus e Scientifc Eletronic Library Online (SCIELO).

A busca procedeu-se no mês de agosto e setembro de 2021 e a delimitação temporal ocorreu nos últimos 6 anos de 2015 a 2021. Os critérios de inclusão serão: artigos na íntegra, acesso gratuito, disponível on-line, idiomas em inglês, espanhol e português.

Como critério de exclusão: teses, dissertações, estudos de revisão, estudos duplicados, capítulos de livros, reportagens, notícias e editoriais.

Após a leitura dos 44 artigos na íntegra, dos quais somente 10 preencheram adequadamente os critérios de inclusão, sendo assim, selecionados para esta revisão integrativa. A descrição dos artigos está apresentada no Figura 1.

Além disso, buscaram-se dados no manual disponível na internet do Ministério da Saúde com conteúdo na cartilha para tratamento de emergência das queimaduras para orientar as equipes de assistência, em âmbito nacional, em situação de urgência e emergência para o tratamento a pacientes portadores de queimaduras (BRASIL, 2012). 
Figura 1 - Fluxograma de descrição dos artigos, encontrados, excluídos e selecionados de acordo com cada base de dados.

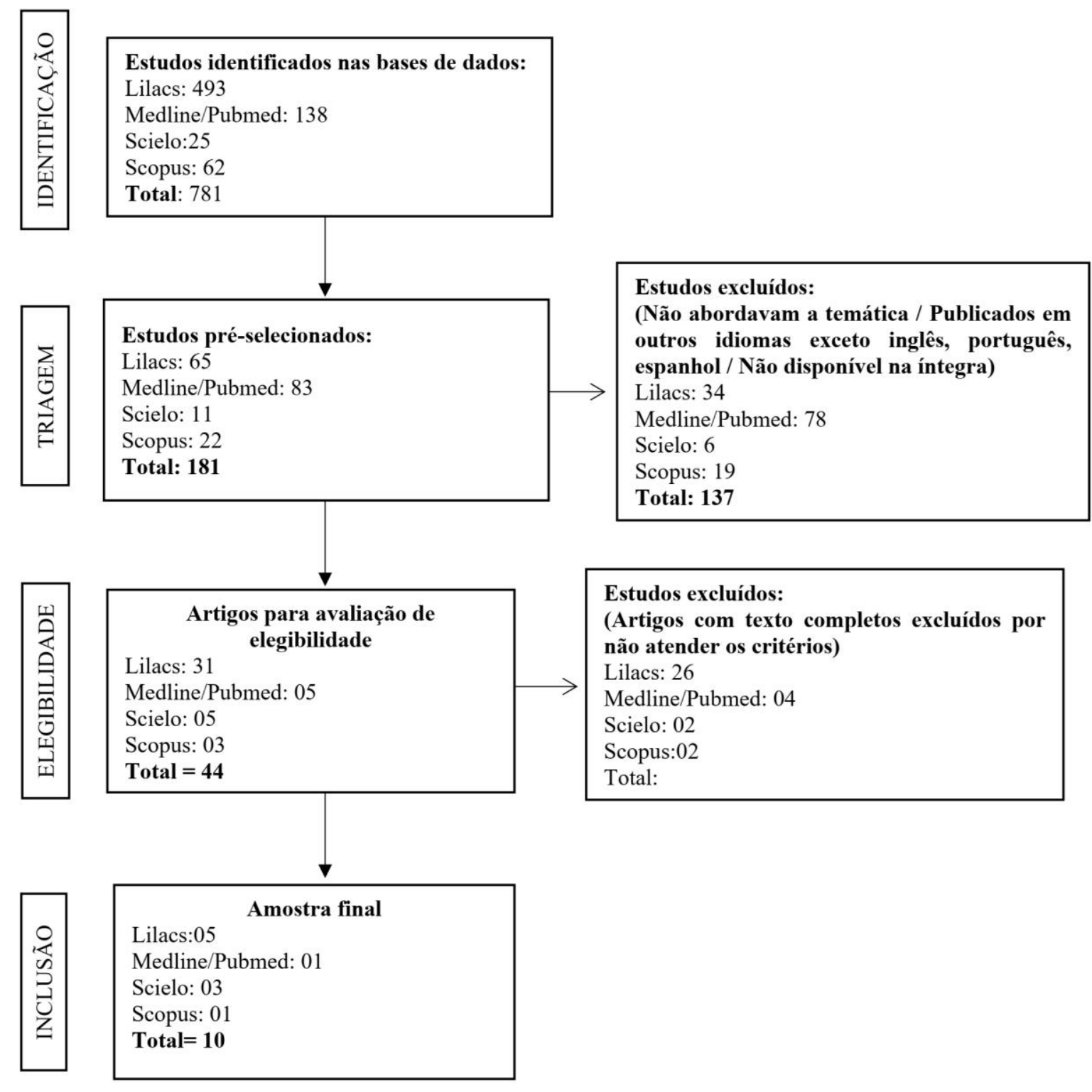

Fonte: Autores.

\section{Resultados}

Quanto à caracterização dos 10 estudos selecionados, o número de publicações por ano foi: 2016: dois, 2017: dois, 2018: um, 2019: dois, 2020: dois e 2021: um (até a data da busca). Em relação ao país em que foram desenvolvidos os estudos: Brasil (8), Estados Unidos da América (1) e África do Sul (1). O idioma predominante nas publicações foi o português (8) e seguido do inglês (2). O Quadro 1 apresenta o autor, ano de publicação, país, título e objetivo dos estudos selecionados. 
Quadro 1 - Caracterização dos estudos acerca do atendimento a crianças vítimas de queimaduras, segundo: ano de publicação, país, título e objetivo, Brasil, 2021.

\begin{tabular}{|c|c|c|c|c|}
\hline Autor & Ano & País & Título & Objetivo \\
\hline Caldas, et al., & 2020 & Brasil & $\begin{array}{l}\text { Sentimentos da enfermagem frente } \\
\text { ao paciente pediátrico sobrevivente } \\
\text { de queimaduras: Uma revisão } \\
\text { narrativa. }\end{array}$ & $\begin{array}{l}\text { Descrever os sentimentos dos profissionais de } \\
\text { enfermagem que atuam frente a crianças vítimas de } \\
\text { queimaduras. }\end{array}$ \\
\hline $\begin{array}{l}\text { Júnior, Azevedo, } \\
\text { Crepaldi, }\end{array}$ & 2017 & Brasil & $\begin{array}{l}\text { Comunicação entre equipe de } \\
\text { saúde, família, criança em unidade } \\
\text { de queimados. }\end{array}$ & $\begin{array}{l}\text { Compreender o processo de comunicação entre } \\
\text { equipe de saúde-família-criança em uma unidade de } \\
\text { queimados. }\end{array}$ \\
\hline Fujisawa et al., & 2016 & Brasil & $\begin{array}{l}\text { Perfil epidemiológico de crianças e } \\
\text { adolescentes vítimas de } \\
\text { queimaduras admitidos em centro } \\
\text { de tratamento de queimados. }\end{array}$ & $\begin{array}{l}\text { Estabelecer o perfil epidemiológico de crianças e } \\
\text { adolescentes atendidas em Centro de Tratamento de } \\
\text { Queimados (CTQ). }\end{array}$ \\
\hline $\begin{array}{l}\text { Queiroz, } \\
\text { Barreto, Lima, }\end{array}$ & 2019 & Brasil & $\begin{array}{l}\text { Crianças vítimas de queimaduras } \\
\text { hospitalizadas em centro de } \\
\text { referência de Fortaleza-Ceará em } \\
2017 .\end{array}$ & $\begin{array}{l}\text { Determinar o perfil das crianças na faixa etária entre } \\
1 \text { e } 12 \text { anos hospitalizadas no centro de referência } \\
\text { para tratamento de queimados em Fortaleza-Ceará no } \\
\text { período de janeiro a dezembro de } 2017\end{array}$ \\
\hline Barros et al., & 2019 & Brasil & $\begin{array}{l}\text { Estudo epidemiológico de } \\
\text { queimaduras em crianças atendidas } \\
\text { em hospital terciário na cidade de } \\
\text { Campo Grande/MS }\end{array}$ & $\begin{array}{l}\text { Descrever o perfil epidemiológico de crianças e } \\
\text { adolescentes vítimas de queimaduras internadas em } \\
\text { uma unidade, referência no tratamento de } \\
\text { queimaduras, de um hospital de grande porte, } \\
\text { localizado na cidade de Campo Grande/MS. }\end{array}$ \\
\hline $\begin{array}{l}\text { Lozano, } \\
\text { Potterton }\end{array}$ & 2018 & $\begin{array}{l}\text { África } \\
\text { do Sul }\end{array}$ & $\begin{array}{l}\text { The use of Xbox Kinect }{ }^{\mathrm{TM}} \text { in a } \\
\text { Pediatric Burns Unit }\end{array}$ & $\begin{array}{l}\text { Investigar o efeito do uso do Xbox Kinect } \\
\text { resultados de crianças na Unidade de Queimaduras } \\
\text { Pediátricas (PBU) do Hospital Acadêmico Chris Hani } \\
\text { Baragwanath. }\end{array}$ \\
\hline Armstrong et al., & 2021 & USA & $\begin{array}{l}\text { Epidemiology and trend of US } \\
\text { pediatric burn hospitalizations, } \\
\text { 2003-2016 }\end{array}$ & $\begin{array}{l}\text { descrever a tendência das admissões hospitalares } \\
\text { pediátricas por queimaduras e as características do } \\
\text { paciente e hospitalar das crianças internadas em } \\
2016 \text {. }\end{array}$ \\
\hline Scapin et al., & 2020 & Brasil & $\begin{array}{l}\text { Realidade virtual como tratamento } \\
\text { complementar no alívio da dor em } \\
\text { crianças queimadas }\end{array}$ & $\begin{array}{l}\text { Relatar a utilização da realidade virtual e os seus } \\
\text { efeitos sob parâmetros clínicos, dor e suas } \\
\text { consequências no tratamento de duas crianças } \\
\text { queimadas em um Centro de Tratamento de } \\
\text { Queimados do Sul do Brasil. }\end{array}$ \\
\hline Passos, et al., & 2016 & Brasil & $\begin{array}{lcc}\text { Sentimentos da equipe } & \text { de } \\
\text { enfermagem decorrentes } & \text { do } \\
\text { trabalho com crianças em } & \text { uma } \\
\text { unidade de queimados. } & \end{array}$ & $\begin{array}{l}\text { Descrever os sentimentos da equipe de enfermagem } \\
\text { sobre seu trabalho com crianças da unidade de } \\
\text { queimados do Hospital Regional da Asa Norte. }\end{array}$ \\
\hline Scapin et al., & 2017 & Brasil & $\begin{array}{l}\text { Utilização da realidade virtual no } \\
\text { tratamento de crianças queimadas: } \\
\text { relato de casos. }\end{array}$ & $\begin{array}{l}\text { Relatar a utilização da Realidade Virtual (RV) na } \\
\text { diminuição da intensidade dolorosa durante a troca de } \\
\text { curativo de duas crianças queimadas internadas em } \\
\text { um Centro de Tratamento ao Queimado (CTQ) do } \\
\text { Sul do Brasil. }\end{array}$ \\
\hline
\end{tabular}

Fonte: Autores.

A partir da leitura e da comparação entre os resultados dos estudos, emergiram quatro categorias, organizadas em: potencialidades, demandas e fragilidades no atendimento a crianças vítimas de queimaduras. Essas categorias são apresentadas no Quadro 2. 
Quadro 2 - Competências, demandas e Debilidades identificadas nos estudos selecionados.

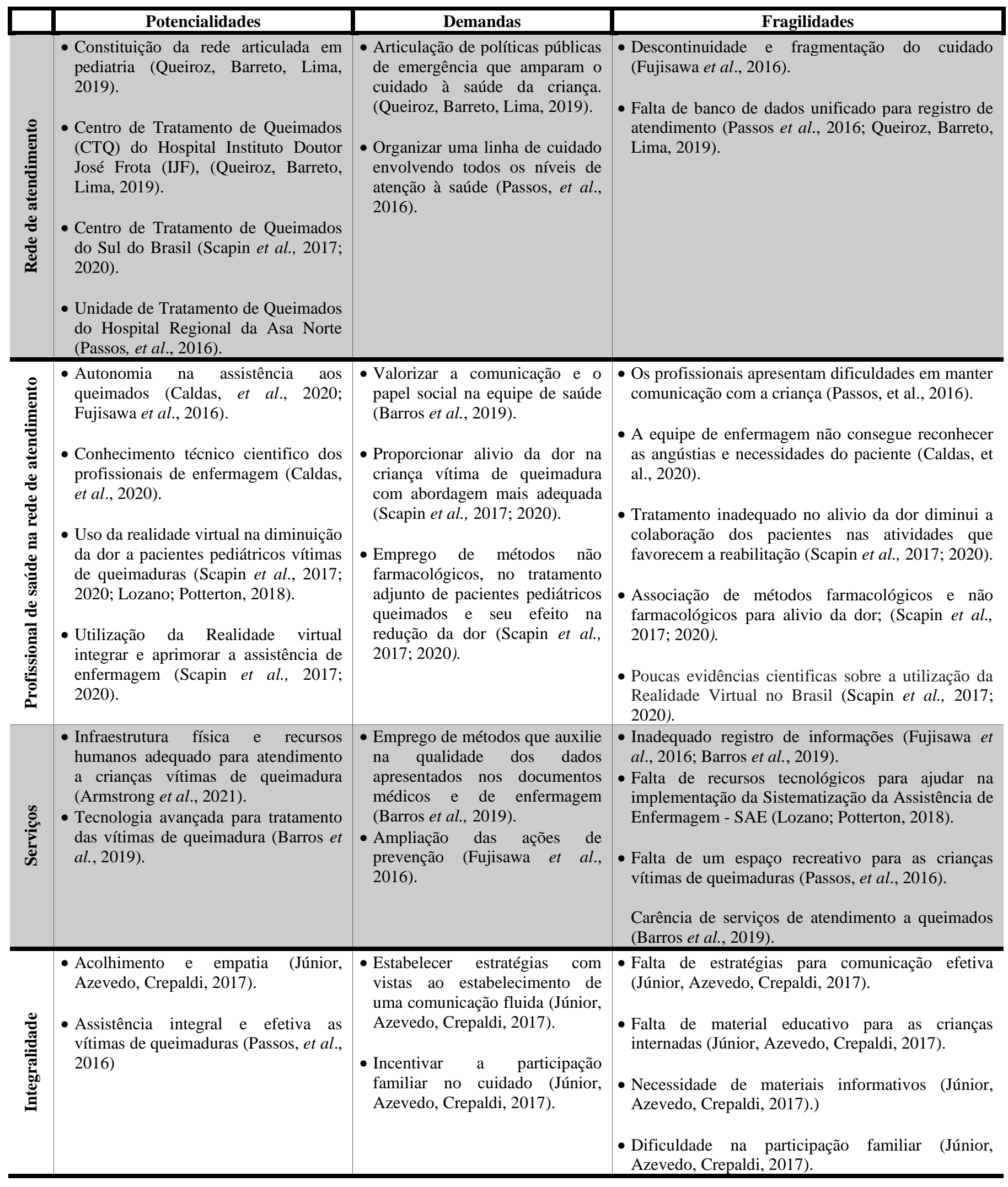




\section{Discussão}

Nos estudos selecionados, algumas questões sobressaem no atendimento a crianças vítimas de queimaduras. A rede de atendimento é uma dessas questões, quando constituída e articulada é uma potencialidade no atendimento (Queiroz, Barreto \& Lima, 2019), outra potencialidade são os centros e/ou unidade de tratamento a queimados (Queiroz, Barreto \& Lima, 2019; Scapin et al., 2020; Passos, et al., 2016). Entretanto, estudos sinalizam fragilidades como a descontinuidade e fragmentação do cuidado (Fujisawa et al., 2016) e a falta de banco de dados unificado para registro de atendimento (Passos et al., 2016; Queiroz, Barreto \& Lima, 2019), gerando assim, demandas de articulação de políticas públicas de emergência que amparam o cuidado à saúde da criança (Queiroz, Barreto \& Lima, 2019) e organizar uma linha de cuidado envolvendo todos os níveis de atenção à saúde (Passos). Essa lacuna, na articulação do serviço e até mesmo a falta de rede traz consequências como a fragmentação do cuidado onde acontece o primeiro atendimento no pronto socorro para posterior atendimento na rede especializada (Cortes \& Padoin, 2016; Cortes, Padoin \& Kinalski, 2017).

Outra categoria encontrada no estudo foi a assistência dos profissionais de enfermagem, que traz como potencialidade a autonomia dos profissionais de enfermagem na assistência aos pacientes queimados (Caldas, et al., 2020; Fujisawa et al., 2016) e o conhecimento técnico científico dos profissionais de enfermagem (Caldas, et al., 2020). Contudo, as dificuldades dos profissionais em manter comunicação com a criança (Passos, et al., 2016) e a falta de reconhecimento pela equipe de enfermagem diante as angústias e necessidades do paciente (Caldas, et al., 2020) e o tratamento inadequado no alívio da dor (Scapin et al., 2020) são fragilidades dos profissionais de enfermagem. Um estudo epidemiológico com objetivo em queimaduras em crianças afirma que entre as necessidades dos profissionais estão as demandas de valorização da comunicação, investimento no papel social na equipe de saúde (Barros et al., 2019) e fornecer o alívio da dor na criança vítima de queimadura com abordagem mais adequada (Scapin et al., 2020).

O enfermeiro deve ter conhecimento científico e técnico suficiente, capacidade de execução da prática de alta qualidade, avaliação holística e integrativa dos pacientes e cooperação com equipes multidisciplinares, pois as queimaduras não devem ser tratadas separadamente das outras partes do corpo. A enfermagem deve sempre manter-se atualizada quanto aos protocolos de cuidado com queimaduras e aos avanços tecnológicos no tratamento (Morais, Oliveira \& Soares, 2008; Leonardi et al., 2010).

Além disso, outra potencialidade da categoria assistência do profissional na rede de atendimento é o uso da realidade virtual na diminuição da dor a pacientes pediátricos vítimas de queimaduras (Scapin et al., 2017; Lozano \& Potterton, 2018). Entretanto, como fragilidades é possível observar a necessidade de associação de métodos farmacológicos com não farmacológicos para alívio da dor, além disso o número de evidências cientificas ainda é escasso sobre a utilização da Realidade Virtual na assistência a pacientes queimados no Brasil com efeito na redução da dor (Scapin et al., 2017; 2020). Portanto o uso de métodos não farmacológicos como realidade virtual na prática clínica pode auxiliar a equipe de enfermagem desde a troca de curativos, a colaboração da criança durante a assistência, como também em reduzir o estresse da equipe.

Nesse contexto, a realidade virtual representa uma terapia complementar promissora, pois tem alcançado resultados positivos na redução da dor e suas consequências (Kipping et al., 2012; Jeffs et al., 2014). Estudos anteriores constataram a eficácia da realidade virtual na redução da dor, especialmente durante processos dolorosos, como trocas de curativos e fisioterapia (Hoffman et al., 2014; Gomez et al., 2017). Fernandes et al., (2012) e Cruvinel et al., (2009), também expuseram o problema da falta de informações no prontuário, dificultando a obtenção de registros e, muitas vezes, informações importantes não são registradas.

Outra categoria observada nos estudos são os serviços, verificou-se como potencialidades, a infraestrutura física e recursos humanos adequado para atendimento a crianças vítimas de queimadura nos Centros de Atendimento ao Paciente Queimado (Armstrong et al., 2021). No entanto é possível identificar fragilidades como o inadequado registro de informações 
(Fujisawa et al., 2016; Barros et al., 2019) e a falta de recursos tecnológicos para ajudar na implementação da Sistematização da Assistência de Enfermagem - SAE (Lozano; Potterton, 2018). Assim é indubitável observar como demanda a necessidade de melhoria da qualidade dos dados apresentados nos registros de assistência dos profissionais de enfermagem (Barros et al., 2019).

Entende-se que uma evolução organizada nos prontuários com descrições detalhada das condutas melhora assistência, pois problemas de omissão de informações em prontuários, resulta na dificuldade em obter-se os registros de qualidade para intervenções futuras, muitas vezes, as informações importantes acabam não sendo documentadas (Fernandes et al.,2012; Costa et al., 2012).

Ainda referente a categoria serviços, foi encontrado também como potencialidade o uso de tecnologia avançada para tratamento das vítimas de queimadura (Barros et al., 2019). Contudo, nessa categoria quando levamos em consideração a estrutura local de atendimento a crianças vítimas de queimadura verificou a importância de um espaço recreativo a esses pacientes (Passos, et al., 2016) e a carência de serviços especializados no atendimento a queimados (Barros et al., 2019), trazendo como demanda a ampliação das ações de prevenção e promoção (Fujisawa et al., 2016).

Durante o processo de hospitalização, muitas vezes as crianças ficam frustradas com a cirurgia invasiva e qualquer ferramenta de distração durante o processo de tratamento torna-se muito importante, pois torna o ambiente hospitalar mais tranquilo e harmonioso. Nesse sentido, a brinquedoteca pode auxiliar essas crianças na distração e no desenvolvimento, seja no crescimento pedagógico como na recuperação hospitalar, uma vez que a hospitalização alterou todos os seus hábitos, e a falta de tais recursos torna o tratamento mais difícil (Weedon \& Potterton, 2011).

Quando leva em consideração as medidas preventivas que devem ser tomadas a crianças vítimas de queimaduras, um estudo o qual avaliou a experiência emocional de enfermeiras ao cuidar de crianças com queimaduras, enfatiza que o maior número de casos vítimas desse trauma vem dos ambientes domésticos. Assim, para a redução de acidentes, é eficaz mobilizar a sociedade por meio do campanhas, bem como aumentar a sensibilidade das famílias e do público em geral por meio de atividades e programas de educação que contemplem o desenvolvimento infantil e ações preventivas. Portanto, o enfermeiro é responsável por agir frente ao processo de educação em saúde e ajudar os familiares durante o tratamento e a recuperação (Hilliard \& O'Neill, 2010; Araújo et al., 2019).

A integralidade traz como potencialidade o acolhimento e empatia dos profissionais. Porém, algumas fragilidades como a falta de estratégias para comunicação efetiva e de material educativo para as crianças internadas são pontos negativos no processo de atendimento, gerando uma necessidade de estabelecer estratégias com vistas ao estabelecimento de uma comunicação fluida (Júnior, Azevedo \& Crepaldi, 2017).

Estudos com mesmo propósito da pesquisa, descreve sobre o preparo físico e psicológico, de um atendimento humanizado ao paciente e familiares. Com objetivo na qualidade e recuperação de alta resolução, diminuição da dor e sofrimento, com uso de procedimentos práticos que auxiliam na autoestima da criança e ajudam pais no processo de sofrimento de culpa. Além disso, Passos et al., (2016), também traz a assistência integral e efetiva as vítimas de queimaduras como potencialidades da integralidade. Contudo, considerou como fragilidade a dificuldade na participação familiar, resultando assim como demanda o incentivo a participação familiar no processo do cuidado (Júnior, Azevedo \& Crepaldi, 2017).

Embora a família seja importante quando a criança está em tratamento, todas as ações que a equipe realizará dependerão da aceitação do procedimento por parte desses familiares, já que se não pode esquecer as diferentes culturas e crenças de algumas famílias, portanto, nem todos os tratamentos necessários é realmente realizado ou autorização pelo núcleo familiar da criança, neste sentido, a assistência pode ser dificultada. O enfermeiro é responsável por repassar as informações necessárias sobre o tratamento a esses familiares e cuidadores, e até mesmo à própria criança, a fim de completar a promoção 
da saúde do paciente infantil e prevenir possíveis sequelas, não deixando de lado a manutenção do equilíbrio físico e emocional da criança em estado de tratamento (Brito et al., 2009).

A presente pesquisa destaca o perfil do atendimento de enfermagem a crianças vítimas de queimaduras que em grande parte esses profissionais são surpreendidos pelo sentimento de sofrimento ao cuidar do paciente infantil vítima de queimadura. A equipe de enfermagem que presta assistência à criança vítima de queimadura deve ser antes de tudo autoconfiante para vivenciar situações que possa enfrentar possíveis barreiras, já que se trata de um setor que causa desgaste e em algumas situações o sofrimento destes profissionais pode estar associado ao dos pacientes que estão sendo tratados.

\section{Conclusão}

Aponta-se que esta revisão integrativa apresenta dados fundamentados sobre o perfil do enfermeiro ao atendimento de crianças vítimas de queimaduras. Nos estudos selecionados, identificamos potencialidades, fragilidades e demandas. Ainda que potencialidades, como rede articulada, autonomia na assistência, acolhimento e empatia; existem fragilidades, como falta de estratégias para comunicação efetiva e inadequado registro de informações, que desafiam a concretização do atendimento integral.

Com a presente pesquisa, pode-se observar a importância dos profissionais de enfermagem na assistência as crianças vítimas de queimadura e que os mesmos possuem conhecimento técnico científico e autonomia na assistência. Além disso, os profissionais utilizam tecnologias, como a realidade virtual, na diminuição da dor dos pacientes pediátricos vítimas de queimaduras. Entretanto, percebeu-se a falta de um espaço recreativo para as crianças vítimas de queimaduras e que a equipe de enfermagem não consegue reconhecer as angústias e necessidades do paciente.

Nota-se uma escassez de estudos a respeito do perfil do atendimento aos pacientes queimados. Sugere-se, contudo, que novos estudos sejam realizados, visando o fortalecimento das evidências sobre o perfil do atendimento de enfermagem a crianças vítimas de queimaduras.

A contribuição dessa revisão para a saúde e a enfermagem é a reflexão acerca de elementos que são fundamentais na concretização da eficácia do atendimento e a serem considerados para aprimorar o atendimento de enfermagem a crianças vítimas de queimaduras. Além disso, observou que a enfermagem tem um papel importante no tratamento de pacientes queimados devendo focar no contexto assistencial e educativo.

Todavia o presente estudo proporcionou conhecimento sobre o perfil do atendimento de enfermagem a crianças vítimas de queimaduras, percebendo-se a importância do enfermeiro em se atualizar, aumentando sua bagagem de conhecimento técnico-científico, a fim de proporcionar assistência adequada à criança e sua família, para que, assim, alcance resultado eficaz em tempo reduzido, não deixando de ter uma visão holística e humanizada.

\section{Referências}

Aragão, J. A., Aragão, M. E. C. S., Filgueira, D. M., Teixeira, R. M. P., \& Reis, F. P. (2012). Estudo epidemiológico de crianças vítimas de queimaduras internadas na Unidade de Tratamento de Queimados do Hospital de Urgência de Sergipe. Revista Brasileira de Cirurgia Plástica, $27,379-382$. https://www.scielo.br/j/rbcp/a/HRvcDJz5Q8y36L5Fw5X9hBf/?format=pdf\&lang=pt

Araújo, C. M., Almeida, F. A., Caetano, A. B., \& Gularte, J. S. (2019). Incidência das internações por queimaduras em crianças no Brasil durante o período de 2008 a 2017. e-Scientia, 12(1), 9-17. https://revistas.unibh.br/dcbas/article/view/2655

Armstrong, M., Wheeler, K. K., Shi, J., Thakkar, R. K., Fabia, R. B., Groner, J. I., \& Xiang, H. (2021). Epidemiology and trend of US pediatric burn hospitalizations, 2003-2016. Burns, 47(3), 551-559. https://www.sciencedirect.com/science/article/abs/pii/S0305417920303879

Barros, L. A. F., Da-Silva, S. B. M., Maruyama, A. B. A., Gomes, M. D., Muller, K. D. T. C., \& do Amaral, M. A. D. O. (2019). Estudo epidemiológico de queimaduras em crianças atendidas em hospital terciário na cidade de Campo Grande/MS. Rev. bras. queimaduras, 71-77. https://pesquisa.bvsalud.org/controlecancer/resource/pt/biblio-1119494?src=similardocs

Bergamasco, E. C., Rossi, L. A., Carvalho, E. C. D., \& Dalri, M. C. B. (2004). Diagnósticos de medo e ansiedade: validação de conteúdo para o paciente queimado. Revista Brasileira de Enfermagem, 57, 170-177. https://www.scielo.br/j/reben/a/RMpNLBZvMfPYv5N4cgVnWZS/abstract/?lang=pt 
Brasil. Ministério da Saúde, Secretaria de Atenção à Saúde, \& Departamento de Atenção Especializada. (2012). Cartilha para tratamento de emergência das queimaduras. https://bvsms.saude.gov.br/bvs/publicacoes/cartilha_tratamento_emergencia_queimaduras.pdf

Brito, M. E. M., de Castro Damasceno, A. K., da Costa Pinheiro, P. N., \& de Souza Vieira, L. J. E. (2010). A cultura no cuidado familiar à criança vítima de queimaduras. Revista Eletrônica de Enfermagem, 12(2), 321-5. http://www.fen.ufg.br/revista/v12/n2/v12n2a14.htm

Caldas, G. R. F., da Silva, J. W. L., dos Santos, L. S. A., de Sousa, B. S., Torres, L. S., \& da Silva, C. R. L. (2020). Sentimentos da enfermagem frente ao paciente pediátrico sobrevivente de queimaduras: Uma revisão narrativa. Rev Bras Queimaduras, 19(1), 00. http://rbqueimaduras.org.br/details/506

Cortes, L. F., \& Padoin, S. M. D. M. (2016). Intentionality of the action of caring for women in situations of violence: contributions to Nursing and Health. Escola Anna Nery, 20. https://www.scielo.br/j/ean/a/b8Yz6Yvh5tskjfFrnrgWnWv/abstract/?lang=en

Cortes, L. F., Padoin, S. M. D. M., \& Kinalski, D. D. F. (2017). Instruments for articulating the network of attention to women in situation of violence: collective construction. Revista gaúcha de enfermagem, 37. https://www.scielo.br/j/rgenf/a/XVjxbntkGsXSdfPjmPmXZ9w/abstract/?lang=en

Cruvinel, C. A. M., de Sá, D. M., Padovese, S. M., \& Sinval, S. (2009). Estudo epidemiológico de queimaduras atendidas nas Unidades de Atendimento Integrado de Uberlândia-MG entre 2000 a 2005. Revista Brasileira de Queimaduras, 8(1), 18-22. http://www.rbqueimaduras.com.br/details/7

Fernandes, F. M. F. D. A., Torquato, I. M. B., Dantas, M. S. D. A., Pontes Júnior, F. D. A. C., Ferreira, J. D. A., \& Collet, N. (2012). Queimaduras em crianças e adolescentes: caracterização clínica e epidemiológica. Revista Gaúcha de Enfermagem, 33, 133-141. https://www.scielo.br/j/rgenf/a/5k3B8mV386prMJXPTp6m8zM/abstract/?lang=pt

Fujisawa, M. A. T., Valenciano, P. J., Itakussu, E. Y., Kakitsuka, E. E., Hoshimo, A. A., Trelha, C. S., \& Shizuko, D. (2016). Perfil epidemiológico de crianças e adolescentes vítimas de queimaduras admitidos em centro de tratamento de queimados. Revista Brasileira de Queimaduras, 15(2), 74-79. http://www.rbqueimaduras.com.br/details/297/pt-BR/perfil-epidemiologico-de-criancas-e-adolescentes-vitimas-de-queimaduras-admitidos-em-centro-detratamento-de-queimados

Gomez, J., Hoffman, H. G., Bistricky, S. L., Gonzalez, M., Rosenberg, L., Sampaio, M., \& Linehan, M. M. (2017). The use of Virtual Reality Facilitates Dialectical Behavior Therapy "Observing Sounds and Visuals" mindfulness skills training exercises for a Latino patient with severe burns: a case study. Frontiers in psychology, 8, 1611. https://www.frontiersin.org/articles/10.3389/fpsyg.2017.01611/full

Gurgel, A. K. C., \& Monteiro, A. I. (2016). Domestic accident prevention for children: perceived susceptibility by the caregivers. Revista de Pesquisa Cuidado é Fundamental Online, 8(4), 5126-5135. https://www.redalyc.org/pdf/5057/505754107028.pdf

Hilliard, C., \& O'Neill, M. (2010). Nurses' emotional experience of caring for children with burns. Journal of clinical nursing, 19(19-20), 2907-2915. https://doi.org/10.1111/j.1365-2702.2009.03177.x

Hoffman, H. G., Meyer III, W. J., Ramirez, M., Roberts, L., Seibel, E. J., Atzori, B., \& Patterson, D. R. (2014). Feasibility of articulated arm mounted Oculus Rift Virtual Reality goggles for adjunctive pain control during occupational therapy in pediatric burn patients. Cyberpsychology, Behavior, and Social Networking, 17(6), 397-401. https://doi.org/10.1089/cyber.2014.0058

Jeffs, D., Dorman, D., Brown, S., Files, A., Graves, T., Kirk, E., \& Swearingen, C. J. (2014). Effect of virtual reality on adolescent pain during burn wound care. Journal of Burn Care \& Research, 35(5), 395-408. https://doi.org/10.1097/BCR.0000000000000019

Júnior, A. C. L., Azevedo, A. V. S., \& Crepaldi, M. A. (2017). Comunicação entre equipe de saúde, família, criança em unidade de queimados. Psicologia em Estudo, 22(4), 623-634. https://doi.org/10.4025/psicolestud.v22i4.35849

Kipping, B., Rodger, S., Miller, K., \& Kimble, R. M. (2012). Virtual reality for acute pain reduction in adolescents undergoing burn wound care: a prospective randomized controlled trial. Burns, 38(5), 650-657. https://doi.org/10.1016/j.burns.2011.11.010

Leonardi, G. A. L., \& Francisco, D. (2010). Transtorno de estresse pós-traumático em pacientes com sequelas de queimaduras. Revista Brasileira de Queimaduras, 9(3), 105-114. http://www.rbqueimaduras.com.br/details/44/pt-BR

Lozano, E. I., \& Potterton, J. L. (2018). The use of Xbox Kinect ${ }^{\mathrm{TM}}$ in a Paediatric Burns Unit. South African Journal of Physiotherapy, 74(1), 1-7. https://hdl.handle.net/10520/EJC-ee9e605ea

Maciel, M. G. L. M., dos Santos, E. L., Costa, A. B., da Silva, M. R., Oliveira, K. C. P. N., \& de Souza, M. D. P. G. (2018). Causa s de queimaduras em crianças atendidas em um hospital público de Alagoas. Revista Brasileira de Queimaduras, 17(1), 43-49. http://www.rbqueimaduras.com.br/details/416/ptBR/causas-de-queimaduras-em-criancas-atendidas-em-um-hospital-publico-de-alagoas

Morais, G. F. D. C., Oliveira, S. H. D. S., \& Soares, M. J. G. O. (2008). Avaliação de feridas pelos enfermeiros de instituições hospitalares da rede pública. Texto \& Contexto-Enfermagem, 17, 98-105. https://www.scielo.br/j/tce/a/vpfJ5vXCGSqxQ5yv6pr8NDt/?format=pdf\&lang=pt

Nettina, S. M. (1999). Prática de enfermagem. (6a ed.) Guanabara Koogan.

Novaes, F. N. (2003). Primeiro atendimento ao paciente queimado. J. bras. med, 56-62. https://pesquisa.bvsalud.org/portal/resource/pt/li1-357974

Oliveira, W. C. M., \& Sales, C. C. F. (2016). Fatores de risco e medidas de prevenção das queimaduras infantis: revisão integrativa da literatura. Revista Brasileira de Queimaduras, 15(4), 267-273. http://www.rbqueimaduras.com.br/details/325/pt-BR/fatores-de-risco-e-medidas-de-prevencao-das-queimadurasinfantis--revisao-integrativa-da-literatura

Passos, G. R. D. P. C., \& Ninômia, M. A. (2016). Sentimentos da equipe de enfermagem decorrentes do trabalho com crianças em uma unidade de queimados. Revista Brasileira de Queimaduras, 15(1), 35-41. http://www.rbqueimaduras.com.br/details/290/pt-BR/sentimentos-da-equipe-de-enfermagemdecorrentes-do-trabalho-com-criancas-em-uma-unidade-de-queimados 
Prestes, H. D., Morais, I. H., \& Alberto, M. (2015). Perfil dos acidentes por queimaduras em crianças atendidas no Hospital Universitário Evangélico de Curitiba. Revista Brasileira de Queimaduras, 14(4), 268-272. http://www.rbqueimaduras.com.br/details/276/pt-BR/perfil-dos-acidentes-por-queimaduras-emcriancas-atendidas-no-hospital-universitario-evangelico-de-curitiba

Queiroz, J. H. M., Barreto, K. L., \& dos Santos Lima, J. (2019). Crianças vítimas de queimaduras hospitalizadas em centro de referência de Fortaleza-Ceará em 2017. Rev Bras Queimaduras, 18(1), 23-6. https://pesquisa.bvsalud.org/portal/resource/pt/biblio-1100103?src=similardocs

Santos, A. M. R. D., Amorim, N. M. A., Braga, C. H., Lima, F. D. M., Macedo, E. M. A. D., \& Lima, C. F. D. (2011). Vivências de familiares de crianças internadas em um Serviço de Pronto-Socorro. Revista da Escola de Enfermagem da USP, 45, 473-479. https://www.scielo.br/j/reeusp/a/t8qfzgfN7MRXHj3mfH59pzL/?lang=pt\&format=html

Scapin, S., Echevarría-Guanilo, M. E., Fuculo, P. R. B., Tomazoni, A., \& Gonçalves, N. (2020). Realidade virtual como tratamento complementar no alívio da dor em crianças queimadas. Texto \& Contexto-Enfermagem, 29. https://doi.org/10.1590/1980-265X-TCE-2018-0277

Scapin, S. Q., Echevarría-Guanilo, M. E., Fuculo, P. R. B., Martins, J. C., Barbosa, M. D. V., \& Pereima, M. J. L. (2017). Use of virtual reality for treating burned children. Revista brasileira de enfermagem, 70, 1291-1295. https://www.scielo.br/j/reben/a/sgSQMwhknvYpYDnbMXsYnKp/?lang=en

Smeltzer, S. C., \& Bare, B. G. (2002). Brunner \& Sudarth: tratado de enfermagem médico-cirúrgica. (7a ed.) Guanabara Koogan.

Truppel, T. C., Meier, M. J., Calixto, R. D. C., Peruzzo, S. A., \& Crozeta, K. (2009). Sistematização da assistência de enfermagem em unidade de terapia intensiva. Revista brasileira de enfermagem, 62, 221-227. https://www.scielo.br/j/reben/a/J6PzYNjhRrHv36RWZQ8QmJS/?format=pdf\&lang=pt

Vale, E. C. S. D. (2005). Primeiro atendimento em queimaduras: a abordagem do dermatologista. Anais Brasileiros de Dermatologia, 80, 9-19. https://www.scielo.br/j/abd/a/TwnrQGbRB7MJFTr5G9tDmMD/abstract/?lang=pt

Weedon, M., \& Potterton, J. (2011). Socio-economic and clinical factors predictive of paediatric quality of life post burn. Burns, 37(4), 572-579. https://doi.org/10.1016/j.burns.2010.12.002 